\title{
La distribución rango-tamaño en la región fronteriza de Sonora y Arizona
}

\author{
Ignacio Cabrera Fernández \\ Maestría en Urbanización y Desarrollo Socio-económico \\ Universidad de Georgia, E.U.A.
}

\section{Resumen}

Esta presentación aborda el estudio de la formación de las redes regionales y urbanas, que ayudan a explicar el comportamiento de la sociedad en sus distintas etapas históricas. Para el análisis se seleccionó a 21 ciudades del noroeste de México y suroeste de los Estados Unidos de América, de acuerdo a la importancia e impacto que ciudades a ambos lados de la frontera han tenido sobre el desarrollo socioeconómico de la región, el cual se identifica como un sistema urbano-regional. Es importante entender la ciudad como una forma histórica, como una expresión del desarrollo de la sociedad en el territorio, como un clásico producto de ciertas relaciones sociales, pero también como portadora y reproductora de relaciones sociales en sí misma.

Palabras clave: población, crecimiento demográfico, redes regionales y urbanas, curva de crecimiento, influencia capitalista.

\begin{abstract}
This article approaches on the makeup of regional and urban networks that are helpful to explain the behavior of the society during its diverse historical stages. 21 cities from the Northwest of Mexico and the Southwest of the United States were chosen for this analysis, according to its importance and the impact that these cities from both sides of the border exert on the socio-economical development of this region, same that is identified as an urbanregional system. It is important to understand the city as a historic form, as an expression of development of the society in the territory, as a classic product resulting from some social relationships; but also as a holder and reproducer of social relationships on itself.
\end{abstract}

Keywords: population, demographic growth, regional and urban networks, growth curve, capitalist influence. 


\title{
LA DISTRIBUCION RANGO-TAMAÑO.EN LA REGION FRONTERIZA DE SONORA Y ARIZONA*
}

\author{
Ignacio Cabrera Fernández \\ Arquitecto, Maestría en Urbanización \\ y Desarrollo Socio-económico, \\ Universidad de Georgia E.U.A.
}

La región fronteriza del norte de México, enfrenta actualmente difíciles problemas económicos, agravados desde la devaluación de febrero de 1982, así como dificultades sociales y culturales que fundamentalmente obedecen a la alta densidad de población, producto a su vez en gran medida del flujo migratorio, a la insuficiente integración a la economía nacional y a la dependencia respecto a los Estados Unidos de Norteamérica (E.U.A.), todo lo cual se traduce en la ausencia de una estrategia capaz de encauzar el desarrollo regional, el que -como se verá a continuación-más bien ha respondido espontáneamente a las necesidades del vecino país.

La historia de la determinación de los límites de la frontera norte del país tiene sus antecedentes más remotos en las disputas territoriales entre la Nueva España y las colonias inglesas en Norteamérica. Ya desde entonces nuestro país, primero como colonia española y más tarde como república, tendría que enfrentar, casi siempre en forma desventajosa, la constante extensión hacia el Sur del vecino del Norte.

"Es hasta el siglo XIX con el Tratado Adams-Onis (1819), firmado entre Estados Unidos de América y España, cuando se definen los primeros límites de la frontera norte de México" (Sepúlveda, 1976).

Pero después de este tratado se sufrieron pérdidas: primero Texas y más adelante California, Arizona, Nuevo México, Nevada, Utah y parte de Wyoming, Colorado, Kansas y Oklahoma. Se firma así en 1848 el Tratado de Guadalupe-Hidalgo que, por su imprecisión y la política expansionista de E.U.A., no pudo impedir la pérdida en 1853 , de "La Mesilla", quedando definida la nueva frontera a partir de los estados de Baja California, Sonora, Chihuahua, Coahuila, Nuevo León y Tamaulipas.

- Ponencia presentada en la Reunión Nacional para el Desarrollo de la Frontera Norte. Nogales, Son. diciembre 1983. 
El sistema internacional suele ser presentado como una estructura funcional que relaciona unidades independientes entre sí y cuyo poder de negociación es homogéneo. Es decir, el enfoque tradicional afirma la independencia de cada sistema nacional en sus relaciones con el exterior, visualizado a través de su status jurídico-institucional y de su capacidad plena de negociación a escala mundial con uno, varios o todos los demás sistemas nacionales.

Esta visión formal del modo de operar del sistema internacional queda desvirtuada en cuanto éste es analizado más en profundidad. El poder relativo de cada sistema nacional no puede medirse por su "capacidad legal para contratar", que no está discutida, sino por la libertad de acción y los logros obtenidos en el proceso histórico de las interrelaciones.

La igualdad jurídica ya expuesta no se verifica en los niveles del desarrollo económico y social. La existencia de disparidades agudas entre los potenciales económicos de cada sistema nacional es una realidad estadística comprobada. Algunos estudios empíricos informan que el proceso de fuerte desigualdad entre los niveles de más alto y bajo desarrollo socioeconómico se ha mantenido estable en los últimos cincuenta años. Esta verificación estadística es una primera manifestación de que por debajo de las simples relaciones formales de equilibrio internacional subyacen procesos que provocan y conservan una situación de desequilibrio de persistentes efectos en el tiempo.

"Este desnivel reconoce sus raíces en factores existentes en el contexto del sistema internacional de relaciones económicas, sociales y políticas. Tales factores son: el primero, que se vincula con la influencia que posee la adopción del modo capitalista de producción en los inicios del desarrollo industrial moderno; el segundo, derivado del anterior, se relaciona con la desigual distribución de las ventajas derivadas del proceso de intercambio comercial entre los integrantes del sistema internacional; y el tercer factor, también derivado del primeramente citado, radica en la desigual tasa de acumulación de recursos económicos y técnicos por cada sistema nacional" (Rofman, 1975).

Por lo anterior, el modelo que presento intenta abordar el estudio de la formación de las redes regionales y urbanas que explique el comportamiento de la sociedad global en sus distintas etapas históricas.

El estado de Sonora limita con el estado de Arizona (E.U.A.), en los municipios de San Luis Río Colorado, Puerto Peñasco, Caborca, Altar, Sáric, Nogales, Santa Cruz, Cananea, Naco y Agua Prieta, compartiendo una línea fronteriza de $590 \mathrm{~km}$ aproximadamente; los centros de población más importantes a lo largo de la línea son: San Luis Río Colorado, Sonoyta, Nogales, Naco y Agua Prieta. 
El sistema urbano-regional fronterizo está formado por un grupo de componentes -actividades urbanas- y sus interrelaciones dentro de un cierto espacio geográfico.

El sistema urbano-regional abarca muchos subsistemas; cuando ellos mantienen una cierta constancia de sus componentes e interrelaciones en el tiempo, son definidos como estructuras. "Las estructuras son las características principales de un sistema por el cual este adquiere su contenido básico. Mientras que el sistema urbano puede cambiar cuantitativamente en el tiempo, los cambios estructurales implican transformaciones cualitativas dentro del sistema y en sus características básicas". (Godelier, 1967). La dimensión de la estructura será dividida por los propósitos de este trabajo en indicadores económicos, sociales y políticos, considerando la interacción permanente entre ellos dentro del sistema urbano total y que a la vez nos ayudará a la identificación de la desigualdad espacial como expresión de desigualdad económica, desigualdad social, y desigualdad política.

El sistema urbano-regional es esencialmente dinámico, las relaciones entre actividades van cambiando en el tiempo, por lo cual la formación de la estructura espacial urbana es un proceso histórico que necesita ser analizado tomando en consideración los diferentes factores que operaron en los diferentes períodos del proceso de urbanización.

He seleccionado algunas ciudades de la región del noroeste de México (Sonora y Sinaloa) y del suroeste de los Estados Unidos de América (Arizona) dado que histórica, cultural, económica y geográficamente funcionan como un sistema, formando a su vez subsistemas.

Las ciudades seleccionadas en el lado mexicano son doce (Hermosillo, Culiacán Ciudad Obregón, Los Mochis, Guaymas, Nogales, San Luis Río Colorado, Navojoa, Guasave, Caborca, Agua Prieta y Cananea), con un rango de población que va de 17,085 a 180,237 habitantes, tomando en cuenta el censo de 1970. Las ciudades seleccionadas del lado norteamericano son nueve (Phoenix, Tucson, Tempe, Mesa, Yuma, Douglas, Casa Grande, Nogales y Bisbee) y con un rango de población que va de 8,328 a 581,562 habitantes durante el mismo año de 1970. Estas ciudades son las que tuvieron y han tenido un importante impacto sobre el desarrollo socioeconómico de la región. El sistema urbano regional cuenta con dos subregiones geográficas bien definidas, las ciudades de la costa y las ciudades localizadas en la región serrana.

El sistema urbano-regional de las 21 ciudades del noroeste de México (Sonora y Sinaloa) y E.U.A. (sur de Arizona) presenta un patrón significativo cuando se aplica la regla rango-tamaño (ver gráfica). El patrón obtenido es significativo porque permite conocer la dinámica del cambio entre el año de 1910 a 1970 y cómo se afectó el sistema de ciudades. La curva obtenida en 
el año de 1910 muestra una distribución convexa que puede significar un desarrollo desarticulado o bien, mezcla de varios sistemas urbanos. Durante el segundo período, en 1940, el sistema de ciudades sufre un fuerte cambio en su patrón de distribución. En este nuevo patrón la gráfica muestra una parte significativa de rango-tamaño distribuido en las ciudades de mayor población el cual teóricamente significa un desarrollo socioeconómico equilibrado, en combinación con curva convexa y primạcía en la mayoría de las ciudades restantes mostrando una desarticulación con el resto de la región. El patrón obtenido en 1940 muestra un importante cambio durante el siguiente período de 1970 donde la curva obtiene mayores características de rango-tamaño, sin mostrar manifestaciones convexas y con una marcada primacía en las más pequeñas y atrasadas ciudades del sistema, con lo cual se muestra cómo el desarrollo socioeconómico de la región tiende hacia un mayor equilibrio dando como resultado un sistema urbano más integrado.

Como se ha mencionado anteriormente, durante el año de 1910 el sistema de ciudades presenta. las características del patrón convexo, típico de la organización urbana con un bajo grado de integración socio-económica del sistema. Los grupos indígenas de la región (yaquis, mayos, pápagos, pimas, seris, etc.) jugaron un importante papel en esta etapa de desarrollo. El actual noroeste de México forma parte, junto con el actual suroeste de E.U.A. que hasta 1848 era territorio mexicano, de una gran área culturalmente similar, sin embargo no estaba económicamente integrada. Spencer (1965) al hablar del noroeste de México dice: "Aunque la frontera suroeste del área cultural del suroeste de los Estados Unidos de América nunca ha sido definida acordemente por los antropólogos, ha sido por un tiempo reconocido que las tribus que forman la cultura del suroeste se extienden en gran parte dentro del noroeste de México".

El bajo grado de integración del sistema heredado de la época colonial, la cual se puede considerar como el inicio de la formación de los principales centros urbanos que nacieron con las misiones establecidas por los jesuitas dentro de las principales áreas indígenas, así como de los pequeños pueblos fundados por los colonos españoles para defenderse de los ataques indios, permitió a los mestizos y población blanca la explotación de la tierra agrícola lo que marcó el inicio del desarrollo de la agricultura cuando algunas "compañías colonizadoras" norteamericanas recibieron la autorización del gobierno de Porfirio Díaz para dividir las zonas agrícolas en parcelas y construir una -red de canales que permitirían la introducción de la agricultura permanente en la región.

De igual forma se logra la consolidación de pequeñas comunidades urbanas (Guaymas, Hermosillo, Nogales) con la introducción del ferrocarril Guaymas-Nogales que se termina en 1882 y después se unen los valles del Yaqui y Mayo, Fuerte y Culiacán, con el objeto de tocar las principales ciudades y regiones de importancia. Principalmente sirvieron como base los objetivos 
Ignacio Cabrera F.

de tipo económico y el deseo de conducir hasta la frontera con los E.U.A aquellos artículos que se exportaban en forma creciente, de interés sobre todo para la industria y al comercio norteamericano.

Por otro lado, a la región serrana la une la vida rural con ausencia de ciudades, inexistencia de una agricultura en grandes áreas, aislamiento casi completo de algunas porciones en lo más alejado de las serranías, y atraso social, económico y cultural de los relativamente escasos habitantes.

El tipo y nivel de vida es palpablemente superior en la región serrana dedicada a la minería, ya que el comercio fronterizo, el desarrollo de la minería del cobre y de la ganadería, permitió un importante desarrollo en ambos lados de la frontera, en donde nuevas comunidades mostraron repentino crecimiento de población (Bisbee, Cananea, Douglas, Agua Prieta).

Durante el segundo período hasta 1940, el gran desarrollo material alcanzado por el Noroeste, se relaciona sin duda a varios hechos ocurridos durante el período postrevolucionario y a los años posteriores a la gran crisis del capitalismo durante los años 1929-33. En primer lugar, con el reparto agrario que el gobierno del general Cárdenas realizó en el año de 1937, en donde se introdujo el ejido de propiedad comunal al repartir parte de los latifundios entre los campesinos, a la vez que consolidó a la nueva élite postrevolucionaria.

En segundo lugar, la ampliación del sistema carretero y el desarrollo del ferrocarril permitió una mejor intercomunicación entre las ciudades de la costa. Esta mejor comunicación entre las ciudades amplió el mercado interno y del comercio con los E.U.A., lo que permitió un crecimiento muy acelerado de la agricultura, ganadería y pesca al tener básicamente una orientación para la exportación, a la vez que se logró un incremento en las actividades de servicio en las principales ciudades del sistema.

Otro aspecto importante fue el inicio de la industrialización en la región, el cual incrementó y concentró las actividades económicas en los mayores centros urbanos, dando como consecuencia un rápido crecimiento de la población en ciudades como Phoenix, Tucson, Culiacán, Los Mochis y Hermosillo. Durante este mismo período surgieron problemas económicos en la región serrana (Cananea, Bisbee, Douglas), que eran los principales centros mineros. La caída en el mercado internacional de los precios del cobre y de otros minerales afectó la economía local; por otro lado, los centros mineros mexicanos fueron también afectados por el impacto del período postrevolucionario, en donde el gobierno mexicano inició el proceso de expropiación de las compañ 1as norteamericanas, como fue el caso de la Moctezuma Cooper Company en Cananea y otros lugares. Lo anterior fue una verdadera catástrofe económica para la región, además de que quedó aislada al tener un pobre sistema de comunicaciones y transportes con el resto de la región, quedando atrasada en relación al resto de las ciudades. 
Durante el último período analizado, de 1940 a 1970 , la región adquiere una homogeneidad en cuanto a la especialización en la actividad agrícola, con crecimiento dependiente del exterior por estar su producción agrícola orientada básicamente hacia la exportación; paralelamente, se ha obtenido un desarrollo en las comunicaciones y en los transportes, así como en la infraestructura agrícola, como parte de los soportes materiales. Se nota la formación de un sistema de ciudades que constituyen en polos de desarrollo industrial, comercial, de transportes, cultural y administrativo, el cual permitió a estos centros urbanos obtener características de rango-tamaño, en contraste con las manifestaciones convexas y marcada primacía en las más pequeñas $\mathrm{y}$ atrasad as ciudades del sistema.

El sistema urbano-regional obtuvo una distribución más continua de ciu. dades con base en el modelo de rango-tamaño. Como se puede apreciar en la gráfica, existe una tendencia en general hacia una distribución urbana "aparentemente" más equilibrada e igualitaria. "Aparentemente", pues como se ve, las principales ciudades del noroeste de México se están integrando y equilibrando con las ciudades del sur de E.U.A. pero la gravedad del problema yace en que los líderes de la jerarquía urbana están de "aquel lado", no de éste: colocando a las ciudades mexicanas en una relación de gran dependencia.

Si observamos la distribución urbana nacional, notaremos la primacía que tiene la ciudad de México con respecto al resto del país, primacía que se está acentuando aún más.

Así es que aquí tenemos dos fenómenos: por un lado la integración de] noroeste mexicano al sur de E.U.A. dentro de un sistema donde guarda una posición desventajosa y por otro lado, la relación de primacía que tiene la ciu. dad de México sobre el Noroeste; es decir, un desarrollo desequilibrado.

Dentro del contexto anterior, si se examina críticamente el desarrollo real de las ciudades en las últimas décadas, y se compara con las propuestas que habían formulado para ellas los correspondientes planes directores, no hay motivo para la satisfacción.

En las ciudades los planes directores no han acertado en acabar con la llamada marginalidad, más bien contribuyeron a acrecentarla. Tales planes urbanísticos sentaron las bases de enormes negocios especulativos con el sue. lo y con la industria de la construcción aprovechando la enorme demanda de los constantes inmigrantes a las principales ciudades.

Es importante entender la ciudad como una forma histórica, como una expresión del desarrollo de la sociedad en el territorio, como un clásico pro. ducto de ciertas relaciones sociales, pero también como portadora y repro. ductora de relaciones sociales ella misma. Hay que entender la ciudad capita. 
Ignacio Cabrera F.

DISTRIBUCION RANGO TAMAÑO

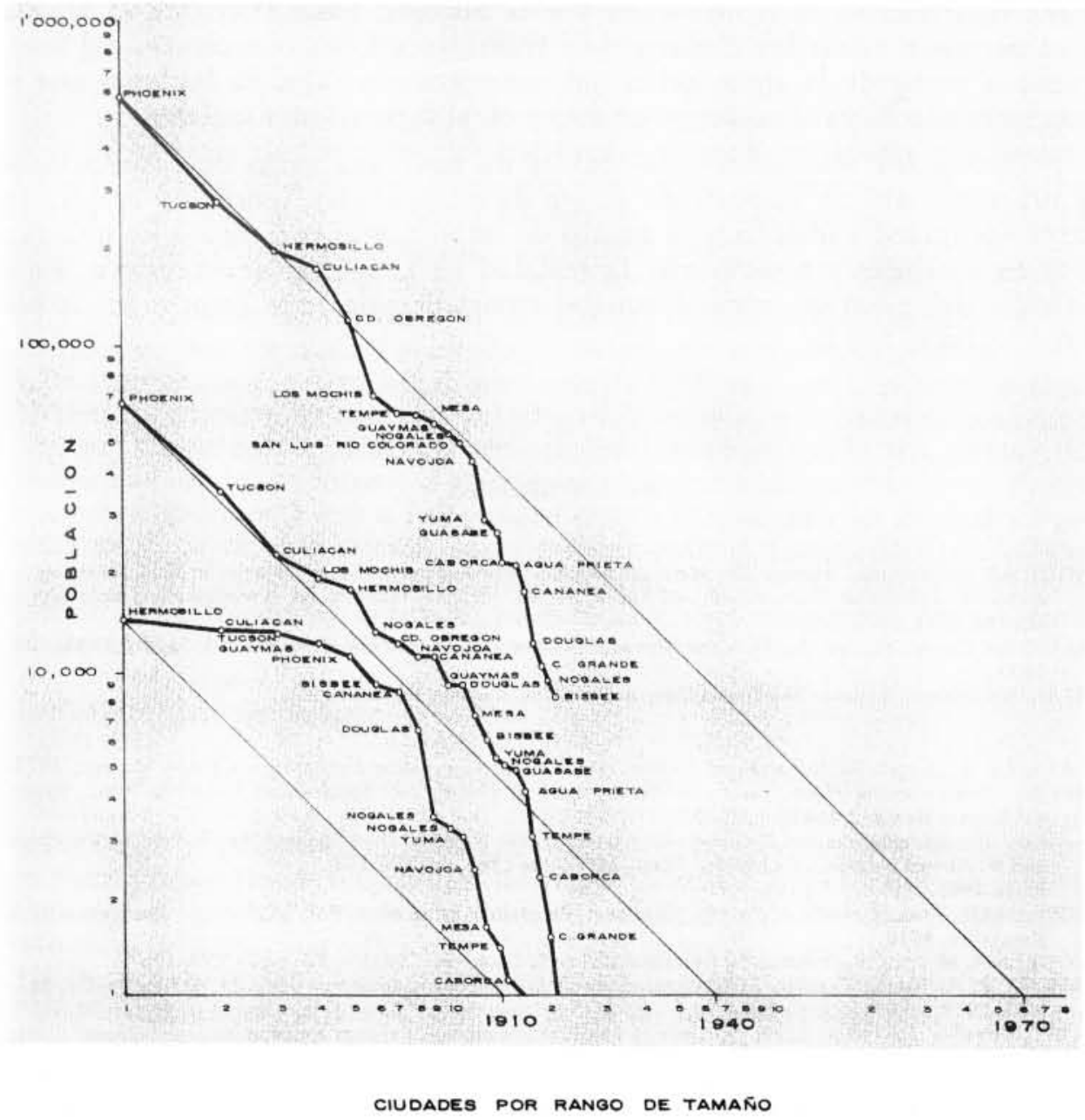


lista con sus características específicas y ubicar los enormes flujos de campesinos a ciertas ciudades como resultado de cambios notables en sus antiguas economías familiares, en su relación con nuevas formas de dominación económica, política e ideológica que los obliga a buscar nuevas posibilidades en otros lados y al otro lado. Por lo tanto, hay que hacer la crítica de las diferentes concepciones del cambio a partir del papel que tales concepciones desempeñan; hacer la crítica de tales concepciones fundándola en categorías cuya cientificidad es corroborada por la historia; basar la crítica en teorías que permitan echar los cimientos de transformaciones conscientes del fenómeno a partir de la apropiación del conocimiento cabal de las leyes que lo han normado hasta el presente, en ésta y otras formaciones sociales.

"Entre los resultados más visibles de toda una gama de concepciones y prácticas está un importante acervo de experiencias, teóricas y empíricas, cuya apropiación dialéctica es asunto de importancia para todos los interesados en aprender y transformar la realidad en la que se desenvuelven, en el sentido del progreso general aunque contradictorio, que caracteriza la historia". (Jury, 1981).

\section{BIBLIOGRAFIA}

AGUILAR Monteverde, Alonso. Un grave problema socio-económico: La concentración de la tierra en el Noroeste. Problemas Estructurales del Subdesarrollo. México: Instituto de Investigaciones Económicas UNAM, 1971, pp. 226-269.

AGUILAR Camin, Héctor. La Frontera Nómada: Sonora y la Revolución Mexicana. Ed. Siglo XXI México, 1977.

ALSBERG, Henry. Arizona: The Grand Canyon State. Ed. Hastings House, 1960.

BASSOLS B., Angel. El Noroeste de México: Un Estudio Geográfico Económico. UNAM, México, 1972. BERRY, Brian, Central Place Studies. A. Bibliography of Theory and Applications Filadelfia, Penn., Regional Science Research Institute, 1965.

- City Size Distribution and Economic Development, en: Regional Development and Planning. J. Friedman and W. Alonso (comps.), Cambridge, Mass., MIT Press 1965, pp. 138-152. Press, 1965.

COPLAMAR. Zona Montaña de Sonora. Coplamar, Programas Integrados. Vol. 26, México: Presidencia de la República, 1978.

GODELIER, Maurice. Racionalidad e Irracionalidad en la Economia. México. Ed. Siglo XXI, 1967.

HEWITT de Alcántara, Cynthia. La Modemización de la Agricultura Mexicana 1940-1970. México, Ed. Siglo XXI, 1978.

JURY, Salvador. La Urbanización en América Latina: Comentarios Críticos a algunas interpretaciones. Copia Xerox. México, 1981.

ROBLES Alejandro. Historia de Sonora. Ed. Nacional, México, 1980.

ROFMAN, Alejandro. Influencia del Proceso Histórico en la Dependencia Externa y en la Estructuración de las Redes Regionales y Urbanas Actuales, Desarrollo Urbano y Regional en América Latina. Ed. Fondo de Cultura Económica, México, 1975, pp. 61-82.

SEPULVEDA César. La Frontera Norte de México. Historia y Conflictos (1762-1975). Ed. Porrúa, México 1976.

SILVERS, Arthur and Pierre Crosson, Rural Development and Urbano-Bound Migration in México. Research Paper R-17, Resources for the Future. Washington, D.C., 1980.

SPENCER, F. Robert, Jesse D. Jennings, et al., The Native Americans. Harper and Row Publisher, N.Y. Pp. 283-336, 1965.

UNIKEL, Luis. El Desarrollo Urbano de México. El Colegio de México, 1976. 